\title{
Comparative Effectiveness and Antibody Responses to Moderna and Pfizer- BioNTech COVID-19 Vaccines among Hospitalized Veterans — Five Veterans Affairs Medical Centers, United States, February 1-September 30, 2021
}

\author{
Kristina L. Bajema, MD ${ }^{1, *}$; Rebecca M. Dahl, MPH ${ }^{1, *}$; Steve L. Evener, MPH ${ }^{1,2}$; Mila M. Prill, MSPH ${ }^{1}$; Maria C. Rodriguez-Barradas, MD ${ }^{3,4}$; \\ Vincent C. Marconi, MD 5,6,7; David O. Beenhouwer, MD ${ }^{8,9}$; Mark Holodniy, MD ${ }^{10,11,12}$; Cynthia Lucero-Obusan, MD ${ }^{10,11}$; Sheldon T. Brown, MD ${ }^{13,14}$; \\ Maraia Tremarelli, MSPH ${ }^{1,15}$; Monica Epperson, $\mathrm{PhD}^{1}$; Lisa Mills, $\mathrm{PhD}^{1}$; So Hee Park ${ }^{1}$; Gilberto Rivera-Dominguez, MD ${ }^{3,4}$; Rosalba Gomez Morones, MD ${ }^{3,4}$; \\ Ghazal Ahmadi-Izadi ${ }^{5}$; Rijalda Deovic, MPH $^{5}$; Chad Mendoza ${ }^{8}$; Chan Jeong ${ }^{8}$; Stephanie J. Schrag, DPhil ${ }^{1}$; Elissa Meites, MD ${ }^{1}$; Aron J. Hall, DVM ${ }^{1}$; \\ Miwako Kobayashi, $\mathrm{MD}^{1}$; Meredith McMorrow, $\mathrm{MD}^{1}$; Jennifer R. Verani, $\mathrm{MD}^{1}$; Natalie J. Thornburg, $\mathrm{PhD}^{1, *}$; \\ Diya Surie, $\mathrm{MD}^{1, *}$; SUPERNOVA COVID-19 Surveillance Group
}

The mRNA COVID-19 vaccines (Moderna and PfizerBioNTech) provide strong protection against severe COVID-19, including hospitalization, for at least several months after receipt of the second dose $(1,2)$. However, studies examining immune responses and differences in protection against COVID-19-associated hospitalization in real-world settings, including by vaccine product, are limited. To understand how vaccine effectiveness (VE) might change with time, $\mathrm{CDC}$ and collaborators assessed the comparative effectiveness of Moderna and Pfizer-BioNTech vaccines in preventing COVID-19-associated hospitalization at two periods (14-119 days and $\geq 120$ days) after receipt of the second vaccine dose among 1,896 U.S. veterans at five Veterans Affairs medical centers (VAMCs) during February 1-September 30, 2021. Among 234 U.S. veterans fully vaccinated with an mRNA COVID-19 vaccine and without evidence of current or prior SARS-CoV-2 infection, serum antibody levels (anti-spike immunoglobulin $\mathrm{G}$ [IgG] and anti-receptor binding domain [RBD] IgG) to SARS-CoV-2 were also compared. Adjusted VE 14-119 days following second Moderna vaccine dose was $89.6 \%(95 \% \mathrm{CI}=80.1 \%-94.5 \%)$ and after the second Pfizer-BioNTech dose was 86.0\% (95\% CI $=77.6 \%-91.3 \%)$; at $\geq 120$ days $\mathrm{VE}$ was $86.1 \%$ (95\% CI $=77.7 \%-91.3 \%)$ for Moderna and 75.1\% (95\% CI $=64.6 \%-82.4 \%)$ for PfizerBioNTech. Antibody levels were significantly higher among Moderna recipients than Pfizer-BioNTech recipients across all age groups and periods since vaccination; however, antibody levels among recipients of both products declined between 14-119 days and $\geq 120$ days. These findings from a cohort of older, hospitalized veterans with high prevalences of underlying conditions suggest the importance of booster doses to help maintain long-term protection against severe COVID-19. ${ }^{\dagger}$

During February 1-September 30, 2021, adults aged $\geq 18$ years hospitalized at five VAMCs (Atlanta, Georgia; the New York City borough of the Bronx; Houston, Texas; Los Angeles, California; and Palo Alto, California) were screened

\footnotetext{
* These authors contributed equally to this report.

${ }^{\dagger}$ https://www.cdc.gov/coronavirus/2019-ncov/vaccines/booster-shot.html
}

for inclusion in this test-negative case-control assessment $(1,3)$. Patients with COVID-19-like illness ${ }^{\S}$ who received a positive SARS-CoV-2 nucleic acid amplification test result were included as case-patients and those with COVID-19-like illness and negative SARS-CoV-2 test results were included as controls' (4).

Data on demographic characteristics, clinical history, and COVID-19 vaccination history were abstracted from electronic health records. ${ }^{* *}$ Full vaccination was defined as receipt of 2 doses of an mRNA COVID-19 vaccine (Moderna or PfizerBioNTech) $\geq 14$ days before the SARS-CoV-2 test. Participants who received only 1 dose of an mRNA COVID-19 vaccine, 2 mRNA doses with receipt of the second dose $<14$ days before the SARS-CoV-2 test, mixed mRNA vaccine products, 3 vaccine doses, or the Janssen (Johnson \& Johnson) COVID-19 vaccine were excluded from the analysis. ${ }^{\dagger \dagger}$

Available residual clinical serum specimens were collected from fully vaccinated hospitalized control patients at all sites and tested at CDC. Specimens were tested using the V-PLEX SARS-CoV-2 panel 2 kit (Meso Scale Diagnostics) ${ }^{\$ S}$ to measure binding IgG levels against three SARS-CoV-2 antigens: the spike protein (anti-spike), the receptor-binding domain of the spike protein (anti-RBD), and the nucleocapsid protein (anti-nucleocapsid). Levels were reported in international binding antibody units (BAU) per milliliter $(\mathrm{mL})$. Control participants with antinucleocapsid antibodies ( $>11.8 \mathrm{BAU} / \mathrm{mL})$, suggesting a prior SARS-CoV-2 infection, were excluded from the final analysis.

\footnotetext{
$\$$ COVID-19-like illness was defined as fever, new or worsened cough or shortness of breath, loss of taste or smell, oxygen saturation on room air $<94 \%$, requirement for noninvasive ventilation or endotracheal intubation with mechanical ventilation, or chest radiograph or computed tomography pulmonary findings consistent with pneumonia.

The test-negative study design is commonly used to assess vaccine effectiveness in observational studies. In this study design, case-patients with symptomatic COVID-19 who test positive for SARS-CoV-2 are compared with controls with the same clinical syndrome who test negative for SARS-CoV-2. This approach is used to reduce bias from differences in health care-seeking behavior and access to testing and care.

** In the Atlanta and Houston VAMCs, COVID-19 vaccination status was further verified through a review of state immunization registries.

$\dagger \dagger$ Sixty-one participants received the Janssen (Johnson \& Johnson) COVID-19 vaccine and were therefore excluded from the analysis.

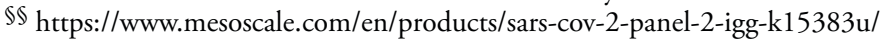


VE to prevent COVID-19-associated hospitalization (calculated as 1 - adjusted odds ratio [aOR] $\times 100)$ 99 was estimated using multivariable logistic regression to compare the odds of full vaccination between case-patients and controls. Models were adjusted for VAMC site, admission date, and age (with the use of cubic splines), sex, and race/ethnicity.*** VE between subgroups was compared using 95\% CIs. In the antibody analysis, pairwise comparisons of median anti-spike IgG and anti-RBD IgG levels using the Wilcoxon rank-sum test and p-values were calculated among participants by age category, vaccine product received, and time since vaccination (14-119 days and $\geq 120$ days after the second vaccine dose). Because vaccines might not elicit a strong immune response ${ }^{\dagger \dagger \dagger}$ in some persons with immunocompromising conditions, ${ }^{\$ \$ \$}$ differences including and excluding this group were examined. Analyses were conducted using SAS (version 9.4; SAS Institute). For all analyses, statistical significance was set at $\mathrm{p}<0.05$. Protocols were reviewed and approved by the VAMC Research and Development Committee at each site. The activity was also reviewed by CDC and conducted consistent with applicable federal law and CDC policy. 999

During February 1-September 30, 2021, a total of 2,329 hospitalized U.S. veterans with COVID-19-like illness met inclusion criteria. After excluding 433 persons with missing data or ineligible vaccination status, ${ }^{* * *} 755$ case-patients and 1,141 controls were included in the analysis. Among these 1,896 patients, $1,758(92.7 \%)$ were male, the median age was 67 years (IQR = 59-75 years), 942 (49.7\%) were Black, and 162 (8.5\%) were Hispanic (Table 1). Effectiveness of the Moderna vaccine was $89.6 \%$ (95\% CI $=80.1 \%-94.5 \%) 14-119$ days after the second vaccine dose and $86.1 \%$ (95\% CI $=77.7 \%-91.3 \%)$ at $\geq 120$ days (Table 2). Effectiveness of the Pfizer-BioNTech vaccine was $86.0 \%(95 \% \mathrm{CI}=77.6 \%-91.3 \%)$ at $14-119$ days and $75.1 \%(95 \% \mathrm{CI}=64.6 \%-82.4 \%)$ at $\geq 120$ days.

Antibody testing was performed on sera available from 259 of $638(40.6 \%)$ fully vaccinated controls. No

\footnotetext{
99 https://www.who.int/publications/i/item/WHO-2019$\mathrm{nCoV}$-vaccine_effectiveness-measurement-2021.1

*** Additional factors were included if they changed the aOR by $\geq 5 \%$ when added individually to the base model.

${ }^{\dagger \dagger \dagger}$ https://www.cdc.gov/coronavirus/2019-ncov/science/science-briefs/fullyvaccinated-people.html

$\$ \$ \$$ Included HIV/AIDS, malignancy, history of solid organ or stem cell transplant, or receipt of immunosuppressive therapy (systemic steroids, chemotherapy, or other immunosuppressive therapy) within 1 month of SARS-CoV-2 test.

99945 C.F.R. part 46.102(l)(2), 21 C.F.R. part 56; 42 U.S.C. Sect. 241(d); 5 U.S.C. Sect. 552a; 44 U.S.C. Sect. 3501 et seq.

**** Included 103 veterans with missing demographic data, vaccination date, or product information; 260 who received only 1 dose of mRNA COVID-19 vaccine or 2 doses $<14$ days before the qualifying SARS-CoV-2 test; three who received mixed mRNA COVID-19 vaccine products; 61 who received the Janssen (Johnson \& Johnson) COVID-19 vaccine; and six fully vaccinated persons who received a third vaccine dose.
}

significant differences in age, sex, or vaccine product received were observed between fully vaccinated controls with and without available sera (Supplementary Table 1, https://stacks.cdc.gov/view/cdc/112103). After excluding

TABLE 1. Characteristics of COVID-19 case-patients and controls* among hospitalized veterans - five Veterans Affairs medical centers, United States, February 1-September 30, 2021

\begin{tabular}{|c|c|c|c|}
\hline \multirow[b]{2}{*}{ Characteristic } & \multicolumn{3}{|c|}{ No. (\%) } \\
\hline & $\begin{array}{c}\text { Total } \\
\mathrm{N}=1,896\end{array}$ & $\begin{array}{l}\text { Case- } \\
\text { patients } \\
\mathrm{n}=755\end{array}$ & $\begin{array}{l}\text { Controls } \\
n=1,141\end{array}$ \\
\hline Male sex & $1,758(92.7)$ & 679 (89.9) & $1,079(94.6)$ \\
\hline Age, median (IQR), yrs & $67(59-75)$ & $63(51-74)$ & $70(62-76)$ \\
\hline $\begin{array}{l}\text { Age group, yrs } \\
18-49 \\
50-64 \\
65-74 \\
75-84 \\
\geq 85\end{array}$ & $\begin{array}{r}241(12.7) \\
551(29.1) \\
621(32.8) \\
334(17.6) \\
149(7.9)\end{array}$ & $\begin{array}{r}166(22.0) \\
238(31.5) \\
189(25.0) \\
114(15.1) \\
48(6.4)\end{array}$ & $\begin{array}{r}75(6.6) \\
313(27.4) \\
432(37.9) \\
220(19.3) \\
101(8.9)\end{array}$ \\
\hline $\begin{array}{l}\text { Race/Ethnicity } \\
\text { Black, non-Hispanic } \\
\text { White, non-Hispanic } \\
\text { Hispanic, any race } \\
\text { Other, non-Hispanic }{ }^{\dagger}\end{array}$ & $\begin{array}{r}942(49.7) \\
748(39.5) \\
162(8.5) \\
44(2.3)\end{array}$ & $\begin{array}{r}377(49.9) \\
277(36.7) \\
82(10.9) \\
19(2.5)\end{array}$ & $\begin{array}{r}565(49.5) \\
471(41.3) \\
80(7.0) \\
25(2.2)\end{array}$ \\
\hline $\begin{array}{l}\text { Resident in long-term care facility } \\
\text { (unknown }=20 \text { ) }\end{array}$ & $114(6.1)$ & $28(3.7)$ & $86(7.6)$ \\
\hline $\begin{array}{l}\text { Study site } \\
\text { Atlanta, Georgia } \\
\text { Bronx, New York City } \\
\text { Houston, Texas } \\
\text { Los Angeles, California } \\
\text { Palo Alto, California }\end{array}$ & $\begin{array}{r}615(32.4) \\
102(5.4) \\
713(37.6) \\
328(17.3) \\
138(7.3)\end{array}$ & $\begin{array}{r}243(32.2) \\
33(4.4) \\
372(49.3) \\
74(9.8) \\
33(4.4)\end{array}$ & $\begin{array}{r}372(32.6) \\
69(6.0) \\
341(29.9) \\
254(22.3) \\
105(9.2)\end{array}$ \\
\hline $\begin{array}{l}\text { Month of admission } \\
\text { Feb-Mar } \\
\text { Apr-Jun } \\
\text { Jul-Sep }\end{array}$ & $\begin{array}{r}451(23.8) \\
442(23.3) \\
1,003(52.9)\end{array}$ & $\begin{array}{l}151(20.0) \\
118(15.6) \\
486(64.4)\end{array}$ & $\begin{array}{l}300(26.3) \\
324(28.4) \\
517(45.3)\end{array}$ \\
\hline COVID-19 fully vaccinated ${ }^{* *}$ & $799(42.1)$ & $161(21.3)$ & $638(55.9)$ \\
\hline $\begin{array}{l}\text { COVID-19 vaccine type among fully } \\
\text { Pfizer BioNTech } \\
\text { Moderna }\end{array}$ & $\begin{array}{l}\text { vaccinated } \\
521(65.2) \\
278(34.8)\end{array}$ & $\begin{array}{r}118(73.3) \\
43(26.7)\end{array}$ & $\begin{array}{l}403(63.2) \\
235(36.8)\end{array}$ \\
\hline $\begin{array}{l}\text { Time between vaccine dose } 2 \text { and } \\
\text { SARS-CoV- } 2 \text { test among fully } \\
\text { vaccinated, median (IQR), days }\end{array}$ & $\begin{array}{r}130 \\
(70-169)\end{array}$ & $\begin{array}{r}157 \\
(125-184)\end{array}$ & $\begin{array}{r}120 \\
(63-163)\end{array}$ \\
\hline Underlying medical condition & & & \\
\hline $\begin{array}{l}\text { Cardiovascular } \\
\text { Atherosclerotic cardiovascular } \\
\text { disease }^{\dagger \dagger}\end{array}$ & $538(29.2)$ & $157(22.0)$ & $381(33.8)$ \\
\hline $\begin{array}{l}\text { Atrial fibrillation } \\
\text { Congestive heart failure }\end{array}$ & $\begin{array}{l}265(14.0) \\
428(22.6)\end{array}$ & $\begin{array}{l}88(11.7) \\
94(12.5)\end{array}$ & $\begin{array}{l}177(15.5) \\
334(29.3)\end{array}$ \\
\hline Hypertension & $1,312(69.2)$ & $478(63.3)$ & $834(73.1)$ \\
\hline Venous thromboembolism & $110(5.8)$ & $41(5.4)$ & $69(6.0)$ \\
\hline $\begin{array}{l}\text { Metabolic } \\
\text { Diabetes } \\
\text { Dyslipidemia } \\
\text { Obesity } \$ \S(\text { unknown = 3) }\end{array}$ & $\begin{array}{l}805(42.5) \\
813(42.9) \\
897(47.4)\end{array}$ & $\begin{array}{l}300(39.7) \\
296(39.2) \\
396(52.6)\end{array}$ & $\begin{array}{l}505(44.3) \\
517(45.3) \\
501(43.9)\end{array}$ \\
\hline $\begin{array}{l}\text { Pulmonary } \\
\text { Asthma } \\
\text { COPD or emphysema } \\
\text { Obstructive sleep apnea }\end{array}$ & $\begin{array}{r}125(6.6) \\
442(23.3) \\
352(18.6)\end{array}$ & $\begin{array}{r}36(4.8) \\
94(12.5) \\
142(18.8)\end{array}$ & $\begin{array}{r}89(7.8) \\
348(30.5) \\
210(18.4)\end{array}$ \\
\hline
\end{tabular}

See table footnotes on the next page. 
$25(9.7 \%)$ control specimens with anti-nucleocapsid antibodies, the analysis included 90 (38.5\%) controls fully vaccinated with the Moderna vaccine (median age $=72$ years; median

TABLE 1. (Continued) Characteristics of COVID-19 case-patients and controls* among hospitalized veterans - five Veterans Affairs medical centers, United States, February 1-September 30, 2021

\begin{tabular}{|c|c|c|c|}
\hline \multirow[b]{2}{*}{ Characteristic } & \multicolumn{3}{|c|}{ No. (\%) } \\
\hline & $\begin{array}{c}\text { Total } \\
N=1,896\end{array}$ & $\begin{array}{c}\text { Case- } \\
\text { patients } \\
\mathrm{n}=755\end{array}$ & $\begin{array}{l}\text { Controls } \\
n=1,141\end{array}$ \\
\hline \multicolumn{4}{|l|}{ Neurologic } \\
\hline Dementia & $111(5.9)$ & $39(5.2)$ & $72(6.3)$ \\
\hline Stroke or transient ischemic attack & $188(9.9)$ & $60(7.9)$ & $128(11.2)$ \\
\hline \multicolumn{4}{|l|}{ Renal } \\
\hline Chronic kidney disease & 372 (19.6) & $122(16.2)$ & $250(21.9)$ \\
\hline End stage renal disease, on dialysis & $82(4.3)$ & $19(2.5)$ & $63(5.5)$ \\
\hline \multicolumn{4}{|l|}{ Liver } \\
\hline Liver disease & $165(8.7)$ & $50(6.6)$ & $115(10.1)$ \\
\hline \multicolumn{4}{|l|}{ Immunocompromising condition } \\
\hline Immunocompromise or therapy & $275(14.9)$ & $64(9.0)$ & $211(18.8)$ \\
\hline \multicolumn{4}{|l|}{ Tobacco use $* * *$} \\
\hline Current & $347(18.3)$ & $91(12.1)$ & $256(22.4)$ \\
\hline Former & $559(29.5)$ & $170(22.5)$ & $389(34.1)$ \\
\hline \multicolumn{4}{|c|}{ No. of hospitalizations during past year (unknown $=45$ ) } \\
\hline 0 & $1,138(61.5)$ & $534(72.7)$ & $604(54.1)$ \\
\hline 1 & 364 (19.7) & $120(16.3)$ & 244 (21.9) \\
\hline$\geq 2$ & 349 (18.9) & $81(11.0)$ & $268(24.0)$ \\
\hline \multicolumn{4}{|l|}{ Outcome } \\
\hline $\begin{array}{l}\text { Intensive care unit admission } \\
\text { (unknown }=10)\end{array}$ & $392(20.7)$ & $179(23.8)$ & $213(18.7)$ \\
\hline Death (unknown = 12) & $108(5.7)$ & $64(8.6)$ & $44(3.9)$ \\
\hline
\end{tabular}

Abbreviations: $\mathrm{COPD}=$ chronic obstructive pulmonary disease; $\mathrm{VAMC}=$ Veterans Affairs medical center.

* Case-patients were defined as patients with COVID-19-like illness (i.e., presence of fever, new or worsened cough or shortness of breath, loss of taste or smell, oxygen saturation on room air $<94 \%$, requirement for noninvasive ventilation or endotracheal intubation with mechanical ventilation, or chest radiograph or computed tomography pulmonary findings consistent with pneumonia) who tested positive for SARS-CoV-2 by nucleic acid amplification test performed within 14 days before admission or during the first 72 hours of hospitalization. Controls were defined as patients with COVID-19-like illness and negative SARS-CoV-2 test results during the same period.

† Included non-Hispanic American Indian and Alaska Native, non-Hispanic Asian and Other Pacific Islander, non-Hispanic multiple races, or non-Hispanic Other race.

$\S$ Included residence before admission to VAMC and non-VAMC nursing facilities as well as other VAMC long-term housing (e.g., domiciliary).

"The Bronx is a borough in New York City.

** COVID-19 vaccination status includes unvaccinated, defined as no receipt of any SARS-CoV-2 vaccine, and fully vaccinated, defined as receipt of both doses of an mRNA (Pfizer-BioNTech or Moderna) $\geq 14$ days before the first SARS-CoV-2 test performed within 14 days before admission or during the first 72 hours of hospitalization.

${ }^{\dagger+}$ Included coronary artery disease, myocardial infarction, peripheral vascular disease, carotid artery stenosis.

$\S \S$ Body mass index $\geq 30 \mathrm{~kg} / \mathrm{m}^{2}$.

११ Included HIV/AIDS, malignancy, history of solid organ or stem cell transplant, or immunosuppressive therapy (systemic steroids, chemotherapy, or other immunosuppressive therapy within 1 month of SARS-CoV-2 test).

*** Tobacco use was defined as smoking of cigarettes, cigars, or pipes. Current use of tobacco was defined as use within the previous 12 months of hospitalization, whereas former use occurred $>12$ months before hospitalization. interval from second dose to serum collection $=75$ days; 24 [26.7\%] with an immunocompromising condition) and 144 $(61.5 \%)$ who were fully vaccinated with the Pfizer-BioNTech vaccine (median age $=73$ years; median interval from second dose to serum collection $=102$ days; 38 [26.4\%] with an immunocompromising condition). Among fully vaccinated Moderna controls, anti-spike IgG levels were higher among persons with sera collected 14-119 days after the second vaccine dose

TABLE 2. Characteristics of case-patients and controls and adjusted effectiveness* of full vaccination ${ }^{\dagger}$ with mRNA COVID-19 vaccines against COVID-19-associated hospitalization among veterans five Veterans Affairs medical centers, ${ }^{\S}$ United States, February 1September 30, 2021

\begin{tabular}{|c|c|c|c|}
\hline \multirow[b]{2}{*}{ Characteristic } & \multicolumn{2}{|c|}{ No./Total no. (\%) } & \multirow[b]{2}{*}{$\begin{array}{l}\text { Adjusted VE } \\
\%(95 \% \mathrm{CI})\end{array}$} \\
\hline & $\begin{array}{c}\text { Case-patients } \\
\text { vaccinated/total }\end{array}$ & $\begin{array}{c}\text { Controls } \\
\text { vaccinated/total }\end{array}$ & \\
\hline Overall & $161 / 755(21.3)$ & $638 / 1,141(55.9)$ & $83.7(78.8-87.5)$ \\
\hline \multicolumn{4}{|l|}{ Age group, yrs } \\
\hline $\begin{array}{l}18-64 \\
\text { Pfizer-BioNTech and } \\
\text { Moderna vaccine } \\
\text { products }\end{array}$ & $33 / 404(8.2)$ & $164 / 388(42.3)$ & $92.2(87.4-95.2)$ \\
\hline Pfizer-BioNTech & $23 / 404(5.7)$ & $86 / 388(22.2)$ & $89.4(80.9-94.1)$ \\
\hline Moderna & $10 / 404(2.5)$ & $78 / 388(20.1)$ & $94.5(88.4-97.4)$ \\
\hline $\begin{array}{l}\geq 65 \\
\text { Pfizer-BioNTech and } \\
\text { Moderna vaccine } \\
\text { products }\end{array}$ & $128 / 351(36.5)$ & $474 / 753(62.9)$ & $75.6(66.2-82.4)$ \\
\hline Pfizer-BioNTech & 95/351 (27.1) & $317 / 753(42.1)$ & $72.9(61.1-81.2)$ \\
\hline Moderna & $33 / 351(9.4)$ & $157 / 753(20.8)$ & $78.6(64.9-86.9)$ \\
\hline \multicolumn{4}{|c|}{ COVID-19 vaccine product $^{\dagger}$} \\
\hline $\begin{array}{l}\text { Pfizer-BioNTech } \\
\text { All periods since } \\
\text { vaccination? }\end{array}$ & $118 / 755(15.6)$ & $403 / 1,141(35.3)$ & $79.8(72.7-85.1)$ \\
\hline 14-119 days & 26/755 (3.4) & $200 / 1,141(17.5)$ & $86.0(77.6-91.3)$ \\
\hline$\geq 120$ days & $92 / 755(12.2)$ & $203 / 1,141(17.8)$ & $75.1(64.6-82.4)$ \\
\hline \multicolumn{4}{|l|}{ Moderna } \\
\hline $\begin{array}{l}\text { All periods since } \\
\text { vaccination" }\end{array}$ & $43 / 755(5.7)$ & $235 / 1,141(20.6)$ & $87.0(80.7-91.2)$ \\
\hline 14-119 days & $12 / 755(1.6)$ & $119 / 1,141(10.4)$ & $89.6(80.1-94.5)$ \\
\hline$\geq 120$ days & $31 / 755(4.1)$ & $116 / 1,141(10.2)$ & $86.1(77.7-91.3)$ \\
\hline \multicolumn{4}{|c|}{ No. of days since vaccination, age group } \\
\hline \multicolumn{4}{|l|}{ 14-119 days } \\
\hline$\geq 18 \mathrm{yrs}$ & $38 / 755(5.0)$ & $319 / 1,141(28.0)$ & $87.8(81.8-91.7)$ \\
\hline $18-64$ yrs & $8 / 404(2.0)$ & $89 / 388(22.9)$ & $95.1(89.1-97.8)$ \\
\hline$\geq 65$ yrs & $30 / 351(8.5)$ & $230 / 753(30.5)$ & $81.2(69.9-88.2)$ \\
\hline \multicolumn{4}{|l|}{$\geq 120$ days } \\
\hline$\geq 18 \mathrm{yrs}$ & $123 / 755$ (16.3) & $319 / 1,141(28.0)$ & $80.0(72.7-85.4)$ \\
\hline $18-64$ yrs & $25 / 404(6.2)$ & 75/388 (19.3) & $89.2(80.8-93.9)$ \\
\hline$\geq 65$ yrs & 98/351 (27.9) & 237/753 (31.5) & $72.9(60.0-81.7)$ \\
\hline
\end{tabular}

Abbreviation: $\mathrm{VE}=$ vaccine effectiveness.

* All nonstratified models adjusted for study site, time (admission date), age, sex, and race/ethnicity. Stratified models exclude adjustment for stratification variable.

+ Includes unvaccinated, defined as no receipt of any SARS-CoV-2 vaccine, and fully vaccinated, defined as receipt of both doses of an mRNA (Pfizer-BioNTech or Moderna) $\geq 14$ days before the first SARS-CoV- 2 test performed within 14 days before admission or during the first 72 hours of hospitalization.

$\S$ The five Veterans Affairs medical centers are located in Atlanta, Georgia; the New York City borough of the Bronx; Houston, Texas; Los Angeles, California; and Palo Alto, California.

" Among fully vaccinated, time since second dose of COVID-19 mRNA vaccine. 
(median = $759 \mathrm{BAU} / \mathrm{mL} ; \mathrm{IQR}=348-2,086 \mathrm{BAU} / \mathrm{mL}$ ) compared with $\geq 120$ days $($ median $=266 \mathrm{BAU} / \mathrm{mL} ; \mathrm{IQR}=133-441$ $\mathrm{BAU} / \mathrm{mL})(\mathrm{p}=0.002)$ (Figure). Anti-spike IgG levels were also higher among fully vaccinated Pfizer-BioNTech controls at 14-119 days after receipt of dose 2 (median $=187 \mathrm{BAU} / \mathrm{mL}$; $\mathrm{IQR}=50-493 \mathrm{BAU} / \mathrm{mL})$ than at $\geq 120$ days $($ median $=62 \mathrm{BAU} / \mathrm{mL}$; $\mathrm{IQR}=25-141 \mathrm{BAU} / \mathrm{mL})(\mathrm{p}=0.001)$. At 14-119 days after the second dose, anti-spike IgG levels were higher among controls fully vaccinated with the Moderna vaccine compared with those who received the Pfizer-BioNTech vaccine among persons aged $18-64$ years $($ median $=612$ versus $340 ; p=0.018$ ) and $\geq 65$ years (median $=792$ versus $152 ; \mathrm{p}<0.001)$. At $\geq 120$ days, anti-spike IgG levels were also higher among controls fully vaccinated with the Moderna vaccine compared with the Pfizer-BioNTech vaccine among persons aged 18-64 years ( median $=267$ versus 106; $\mathrm{p}=0.006$ ) and $\geq 65$ years ( median $=266$ versus $57 ; \mathrm{p}=0.003$ ). Relative differences in anti-RBD IgG levels across groups were similar to differences in anti-spike IgG levels (Supplementary Table 2,_https://stacks.cdc.gov/view/cdc/112104), and differences in anti-SARS-CoV-2 antibody levels were similar across groups with immunocompromised persons included or excluded from the analysis.

\section{Discussion}

Among U.S. veterans hospitalized at five VAMCs during February-September 2021, mRNA COVID-19 vaccines remained effective in preventing COVID-19-associated hospitalizations $\geq 120$ days after receipt of the second dose of Moderna (VE $=86 \%$ ) or Pfizer-BioNTech vaccines $(\mathrm{VE}=75 \%)$. Among recipients of Moderna and PfizerBioNTech vaccines, anti-SARS-CoV-2 spike and RBD IgG levels declined with increasing time since vaccination, although U.S. veterans who received the Moderna vaccine consistently had higher antibody levels compared with recipients of the Pfizer-BioNTech vaccine across age groups and time since vaccination. These findings from a cohort of older, hospitalized veterans with high prevalences of underlying conditions suggest the importance of booster doses to help maintain long-term protection against severe COVID-19.

Although an immune correlate of protection for COVID-19 vaccination has yet to be established, studies have shown a relationship between binding antibody levels, neutralizing antibody levels, and vaccine efficacy in clinical trials $(5,6)$. Pairing antibody levels from the same population in which COVID-19 VE is estimated can inform how changes in humoral immunity relate to real-world protection against

FIGURE. Serum anti-spike and anti-receptor binding domain immunoglobulin G levels* after full vaccination among hospitalized veterans without current or previous SARS-CoV-2 infection ${ }^{\dagger}$ — five Veterans Affairs medical centers, $\$$ United States, February 1-September $30,2021^{\uparrow}$

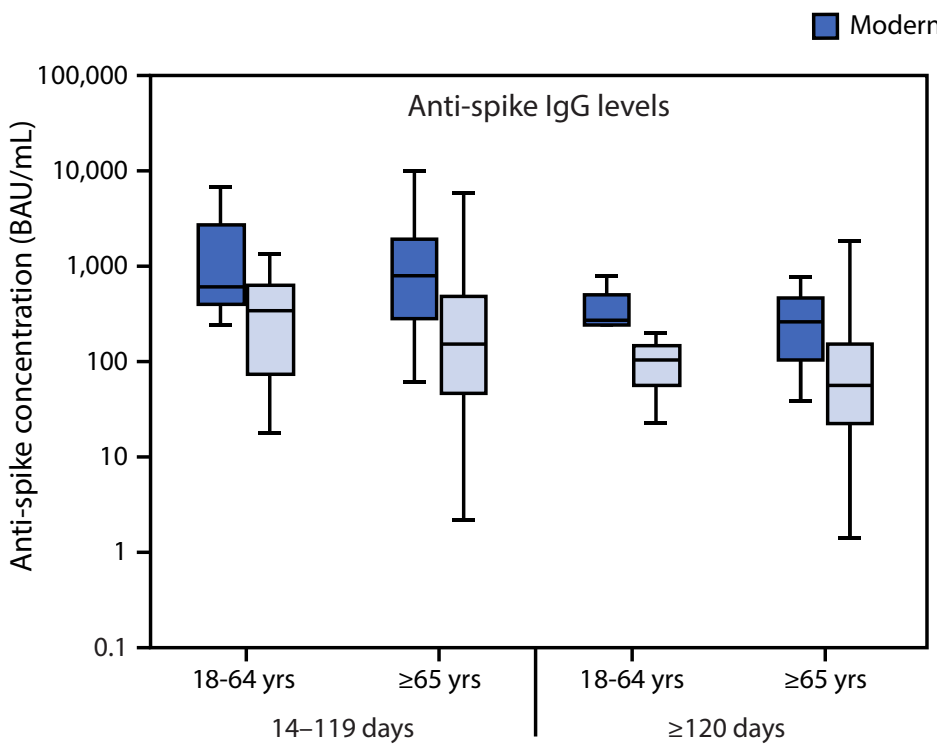

Age group/No. of days since receipt of vaccine dose 2
Pfizer-BioNTech

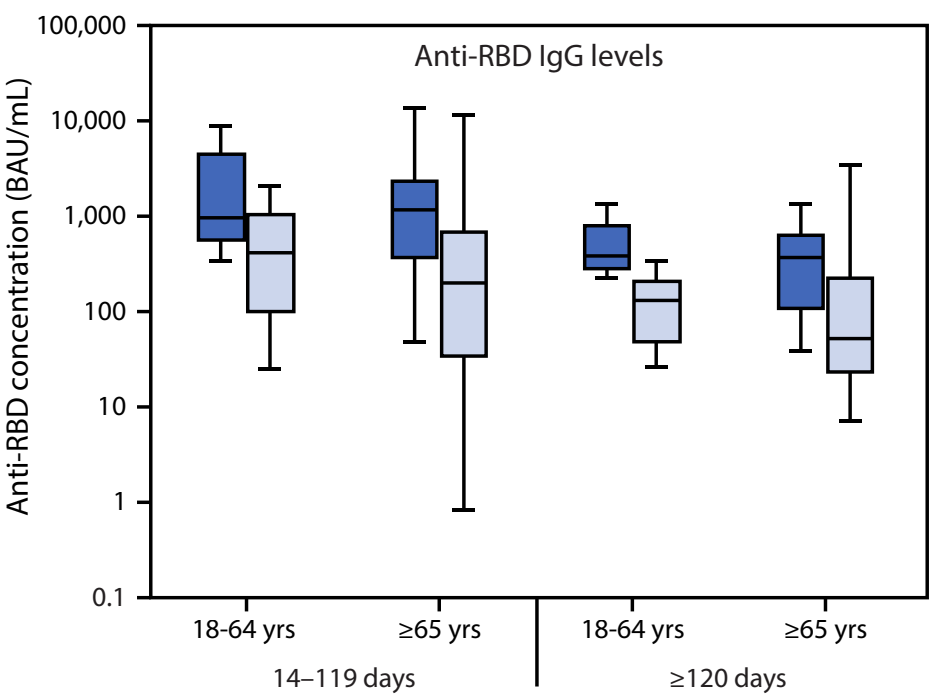

Age group/No. of days since receipt of vaccine dose 2

\footnotetext{
Abbreviations: $\mathrm{BAU}=$ binding antibody units; $\mathrm{lg} \mathrm{G}=$ immunoglobulin $\mathrm{G} ; \mathrm{RBD}=$ receptor binding domain .

* Anti-spike and anti-RBD lgG levels were measured in sera of hospitalized veterans collected at or within 2 days of hospital admission. In these box and whisker plots, the central horizontal line of each box plot represents the median, with the box denoting the IQR, and the whiskers representing $1.5 \times I Q R$.

† Excluded 25 controls with anti-nucleocapsid antibodies $(>11.8 \mathrm{BAU} / \mathrm{mL})$, suggesting a previous SARS-CoV-2 infection.

$\S$ The five Veterans Affairs medical centers are located in Atlanta, Georgia; the New York City borough of the Bronx; Houston, Texas; Los Angeles, California; and Palo Alto, California.

१ Serum specimens collected during March 22-August 31, 2021.
} 


\section{Summary}

What is already known about this topic?

mRNA COVID-19 vaccines are effective in preventing severe COVID-19. Some studies have shown declines in vaccine effectiveness against severe COVID-19 with increasing time since vaccination.

What is added by this report?

During February 1-September 30, 2021, mRNA vaccine effectiveness in preventing COVID-19-associated hospitalizations among U.S. veterans $\geq 120$ days after receipt of the second dose was $86 \%$ for Moderna and $75 \%$ for PfizerBioNTech vaccines. Antibody responses to both vaccines decreased over time. Moderna vaccine recipients had higher antibody levels than did Pfizer-BioNTech recipients.

What are the implications for public health practice?

These findings from a cohort of older, hospitalized veterans with high prevalences of underlying conditions suggest the importance of booster doses to help maintain long-term protection against severe COVID-19.

COVID-19. Although this analysis was not powered to detect small differences in VE by mRNA product as seen in other hospitalized settings ( 7 ), significantly higher post-Moderna vaccination antibody levels compared with Pfizer-BioNTech were observed, which is consistent with findings from other studies $(7,8)$. Potential reasons for this difference include higher antigen content and a longer interval between doses for the Moderna vaccine compared with the Pfizer-BioNTech vaccine (8). Overall, for both vaccine products, antibody levels in this cohort of older U.S. veterans with high prevalences of underlying medical conditions were substantially lower than levels seen among younger, healthy volunteers or health care personnel in other studies $(7,9)$. Consistent with results from studies among younger, healthy persons, antibody levels appeared to wane over time but remained detectable $\geq 120$ days after vaccination $(9,10)$. Although not statistically significant, VE point estimates also declined between 14-119 days and $\geq 120$ days from receipt of second vaccine dose.

The findings in this report are subject to at least four limitations. First, there was insufficient statistical power to detect potential small differences in VE by vaccine product or period since vaccination. Second, it was not possible to assess antibody levels or VE beyond 4 months since receipt of second vaccine dose. Third, residual clinical sera were only available from $41 \%$ of fully vaccinated controls. Finally, binding antibody levels are a surrogate correlate of protection against SARS-CoV-2 and other components of immunity, such as cell-mediated immune responses, were not measured.
Both mRNA COVID-19 vaccines that are approved by the Food and Drug Administration or authorized for use in the United States remain effective against COVID-19-associated hospitalization among U.S. veterans. Antibody levels in this cohort of older persons with high prevalences of underlying medical conditions were lower than those in younger, healthier populations and declined over time. Continued monitoring of the effectiveness of COVID-19 vaccines alongside antiSARS-CoV-2 antibody levels is needed to better understand the duration of protection of these vaccines and the correlation of antibody levels with protection. These findings suggest the importance of booster doses to help maintain long-term protection against severe COVID-19.

\section{Acknowledgments}

Daoling Bi, Cristina Cardemil, Aaron Curns, Fiona Havers, Jefferson Jones, Lindsay Kim, L. Clifford McDonald.

\section{Surveillance Platform for Enteric and Respiratory Infectious Organisms at the VA (SUPERNOVA) COVID-19 Surveillance Group}

Joy Burnette, Atlanta Veterans Affairs Medical Center, Atlanta, Georgia; Gustavo Capo, Atlanta Veterans Affairs Medical Center, Atlanta, Georgia; Lauren Epstein, Atlanta Veterans Affairs Medical Center, Atlanta, Georgia; Julia Gallini, Atlanta Veterans Affairs Medical Center, Atlanta, Georgia; Telisha Harrison, Atlanta Veterans Affairs Medical Center, Atlanta, Georgia; Amy Hartley, Atlanta Veterans Affairs Medical Center, Atlanta, Georgia; Liliana Hernandez, Atlanta Veterans Affairs Medical Center, Atlanta, Georgia; Elena Morales, Atlanta Veterans Affairs Medical Center, Atlanta, Georgia; Nina Patel, Atlanta Veterans Affairs Medical Center, Atlanta, Georgia; Kim Rooney, Atlanta Veterans Affairs Medical Center, Atlanta, Georgia; Tehquin Tanner, Atlanta Veterans Affairs Medical Center, Atlanta, Georgia; Ernest Tate, Atlanta Veterans Affairs Medical Center, Atlanta, Georgia; Ashley Tunson, Atlanta Veterans Affairs Medical Center, Atlanta, Georgia; Alexis Whitmire, Atlanta Veterans Affairs Medical Center, Atlanta, Georgia; Juton Winston, Atlanta Veterans Affairs Medical Center, Atlanta, Georgia; Katherine Elliot, James J. Peters Veterans Affairs Medical Center, Bronx, New York; Ilda Graham, James J. Peters Veterans Affairs Medical Center, Bronx, New York; Diki Lama, James J. Peters Veterans Affairs Medical Center, Bronx, New York; Ismael Pena, James J. Peters Veterans Affairs Medical Center, Bronx, New York; Adrienne Perea, James J. Peters Veterans Affairs Medical Center, Bronx, New York; Guerry Anabelle Perez, James J. Peters Veterans Affairs Medical Center, Bronx, New York; Johane Simelane, James J. Peters Veterans Affairs Medical Center, Bronx, New York; Sarah Smith, James J. Peters Veterans Affairs Medical Center, Bronx, New York; Gabriela Tallin, James J. Peters Veterans Affairs Medical Center, Bronx, New York; Amelia Tisi, James J. Peters Veterans Affairs Medical Center, Bronx, New York; Alonso Arellano Lopez, Michael E. DeBakey Veterans Affairs Medical Center, Houston, Texas; Miguel Covarrubias Gonzalez, Michael E. DeBakey Veterans 
Affairs Medical Center, Houston, Texas; Bashir Lengi, Michael E. DeBakey Veterans Affairs Medical Center, Houston, Texas; Mariana Vanoye Tamez, Michael E. DeBakey Veterans Affairs Medical Center, Houston, Texas; Babak Aryanfar, Veterans Affairs Greater Los Angeles Healthcare System, Los Angeles, California; Ian LeeChang, Veterans Affairs Greater Los Angeles Healthcare System, Los Angeles, California; Anthony Matolek, Veterans Affairs Greater Los Angeles Healthcare System, Los Angeles, California; Aleksandra Poteshkina, Veterans Affairs Greater Los Angeles Healthcare System, Los Angeles, California; Saadia Naeem, Veterans Affairs Greater Los Angeles Healthcare System, Los Angeles, California; Evan Goldin, Veterans Affairs Greater Los Angeles Healthcare System, Los Angeles, California; Madhuri Agrawal, Veterans Affairs Palo Alto Health Care System, Palo Alto, California; Jessica Lopez, Veterans Affairs Palo Alto Health Care System, Palo Alto, California; Theresa Peters, Veterans Affairs Palo Alto Health Care System, Palo Alto, California; Geliya Kudryavtseva, Veterans Affairs Palo Alto Health Care System, Palo Alto, California; Jordan Cates, CDC; Anita Kambhampati, CDC

Corresponding author: Kristina L. Bajema, kbajema@cdc.gov.

${ }^{1}$ CDC COVID-19 Response Team; ${ }^{2}$ Karna, LLC, Atlanta, Georgia; ${ }^{3}$ Michael E. DeBakey Veterans Affairs Medical Center, Houston, Texas; ${ }^{4}$ Department of Medicine, Baylor College of Medicine, Houston, Texas; ${ }^{5}$ Atlanta VA Medical Center, Atlanta, Georgia; ${ }^{6}$ Department of Medicine, Emory University School of Medicine, Atlanta, Georgia; ${ }^{7}$ Department of Global Health, Rollins School of Public Health, Emory University, Atlanta, Georgia; ${ }^{8}$ Veterans Affairs Greater Los Angeles Healthcare System, Los Angeles, California; ${ }^{9}$ Department of Medicine, David Geffen School of Medicine at UCLA, Los Angeles, California; ${ }^{10}$ Veterans Affairs Palo Alto Health Care System, Palo Alto, California; ${ }^{11}$ Public Health Program Office, Department of Veterans Affairs, Washington, DC; ${ }^{12}$ Department of Medicine, Stanford University, Stanford, California; ${ }^{13}$ Department of Medicine, Icahn School of Medicine at Mount Sinai, New York, New York; ${ }^{14}$ James J. Peters Veterans Affairs Medical Center, Bronx, New York, New York; ${ }^{15}$ General Dynamics Information Technology, Falls Church, Virginia.

All authors have completed and submitted the International Committee of Medical Journal Editors form for disclosure of potential conflicts of interest. Vincent C. Marconi reports institutional support to Emory University from the AIDS Clinical Trials Group and the National Institutes of Health (NIH); grants from Lilly, Gilead, ViiV, NIH, and the Veterans Health Administration; payment or honoraria from Medscape, WebMD, ViiV, Integritas, and Lilly; travel support from NIH; and participation on an NIH Data Safety Monitoring Board. Miwako Kobayashi reports support for attending a meeting from the American Veterinary Medical Association. No other potential conflicts of interest were disclosed.

\section{References}

1. Bajema KL, Dahl RM, Prill MM, et al.; SUPERNOVA COVID-19; Surveillance Group; Surveillance Platform for Enteric and Respiratory Infectious Organisms at the VA (SUPERNOVA) COVID-19 Surveillance Group. Effectiveness of COVID-19 mRNA vaccines against COVID-19-associated hospitalization-five Veterans Affairs Medical Centers, United States, February 1-August 6, 2021. MMWR Morb Mortal Wkly Rep 2021;70:1294-9. PMID:34529636 https://doi. org/10.15585/mmwr.mm7037e3

2. Tenforde MW, Self WH, Naioti EA, et al.; IVY Network Investigators; IVY Network. Sustained effectiveness of Pfizer-BioNTech and Moderna vaccines against COVID-19-associated hospitalizations among adultsUnited States, March-July 2021. MMWR Morb Mortal Wkly Rep 2021;70:1156-62. PMID:34437524 https://doi.org/10.15585/mmwr. $\mathrm{mm} 7034 \mathrm{e} 2$

3. Meites E, Bajema KL, Kambhampati A, et al. Adapting the Surveillance Platform for Enteric and Respiratory Infectious Organisms at United States Veterans Affairs Medical Centers(SUPERNOVA) for COVID-19 among hospitalized adults: surveillance protocol. Front Public Health 2021;9:739076. PMID:34778173 https://doi.org/10.3389/ fpubh.2021.739076

4. Jackson ML, Nelson JC. The test-negative design for estimating influenza vaccine effectiveness. Vaccine 2013;31:2165-8. PMID:23499601 https://doi.org/10.1016/j.vaccine.2013.02.053

5. Khoury DS, Cromer D, Reynaldi A, et al. Neutralizing antibody levels are highly predictive of immune protection from symptomatic SARS-CoV-2 infection. Nat Med 2021;27:1205-11. PMID:34002089 https://doi.org/10.1038/s41591-021-01377-8

6. Gilbert PB, Montefiori DC, McDermott A, et al. Immune correlates analysis of the mRNA-1273 COVID-19 vaccine efficacy trial. medRxiv [Preprint posted online August 15, 2021]. https://www.medrxiv.org/ content/10.1101/2021.08.09.21261290v4

7. Self WH, Tenforde MW, Rhoads JP, et al.; IVY Network. Comparative effectiveness of Moderna, Pfizer-BioNTech, and Janssen (Johnson \& Johnson) vaccines in preventing COVID-19 hospitalizations among adults without immunocompromising conditions-United States, March-August 2021. MMWR Morb Mortal Wkly Rep 2021;70:1337-43. PMID:34555004 https://doi.org/10.15585/mmwr.mm7038e1

8. Steensels D, Pierlet N, Penders J, Mesotten D, Heylen L. Comparison of SARS-CoV-2 antibody response following vaccination with BNT162b2 and mRNA-1272. JAMA 2021;326:1533-5. PMID:34459863 https://doi.org/10.1001/jama.2021.15125

9. Laing ED, Weiss CD, Samuels EC, et al. Durability of antibody responses and frequency of clinical and sub-clinical SARS-CoV-2 infection six months after BNT162b2 COVID-19 vaccination in healthcare workers. medRxiv [Preprint posted online October 18, 2021]. https://www. medrxiv.org/content/10.1101/2021.10.16.21265087v1

10. Doria-Rose N, Suthar MS, Makowski M, et al.; mRNA-1273 Study Group. Antibody persistence through 6 months after the second dose of mRNA-1273 vaccine for COVID-19. N Engl J Med 2021;384:2259-61. PMID:33822494 https://doi.org/10.1056/ NEJMc2103916 